
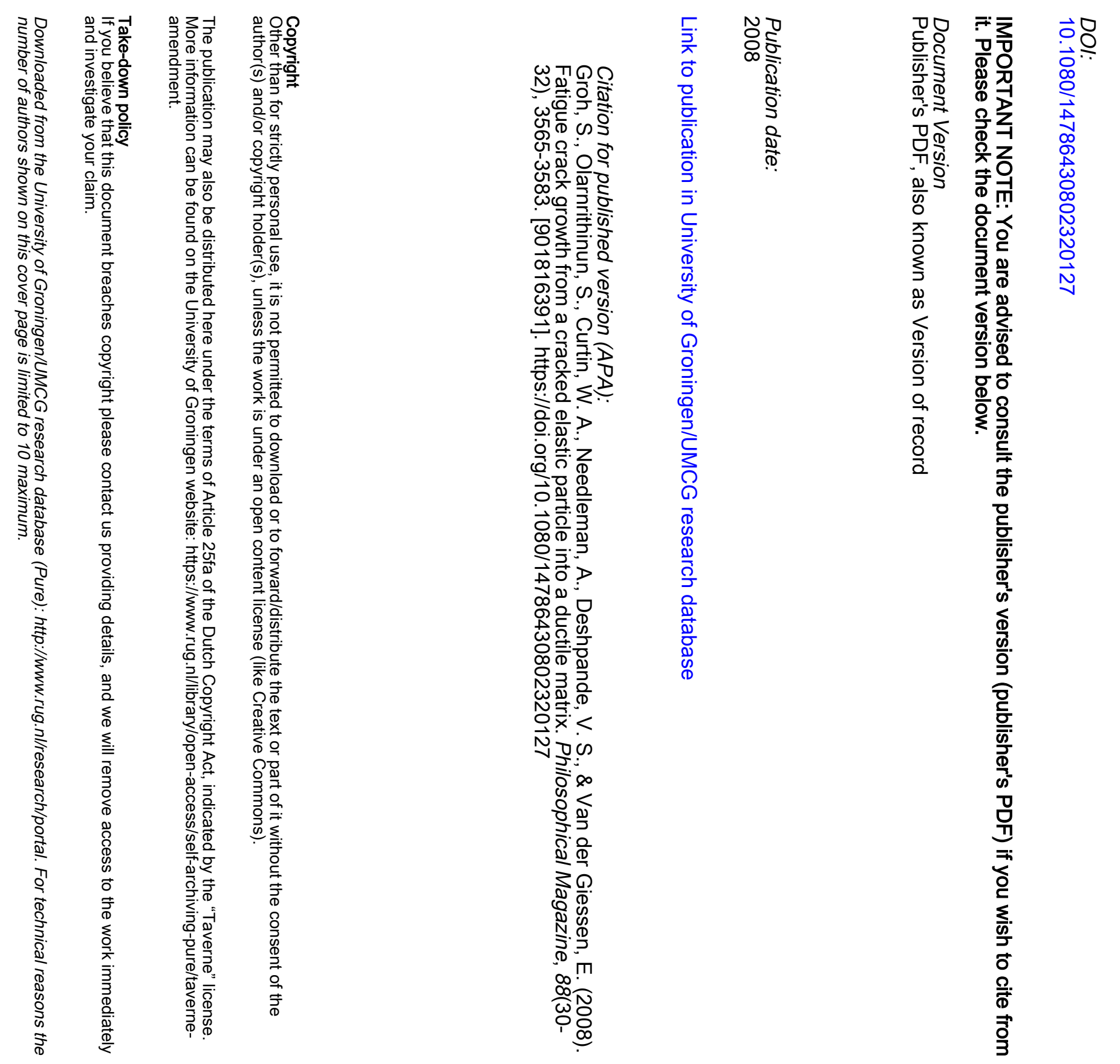

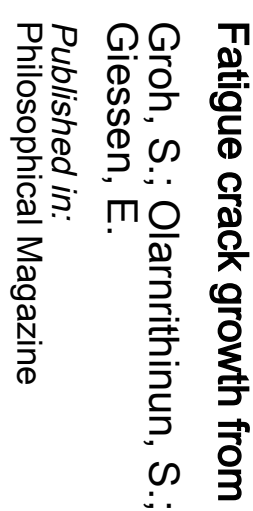

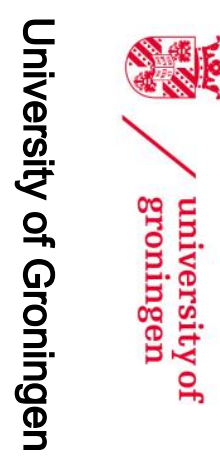

○ ڤ

亭. 옹

$\sum$ هิ

$>\frac{\mathbb{D}}{\mathrm{D}}$

乙

(D)

음

ปั

ᄀ

( )

믕

Фิ 동

प्र

$\begin{array}{ll}3 \\ \frac{0}{0} \\ < & \frac{0}{3} \\ <\end{array}$

o

ఏ

옥

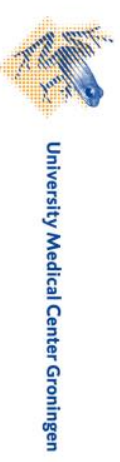




\title{
Fatigue crack growth from a cracked elastic particle into a ductile matrix
}

\author{
S. Groh ${ }^{\mathrm{a}}$, S. Olarnrithinun ${ }^{\mathrm{a}}$, W.A. Curtin ${ }^{\mathrm{a} *}$, A. Needleman ${ }^{\mathrm{a}}$, V.S. Deshpande ${ }^{\mathrm{b}}$ \\ and E. Van der Giessen ${ }^{\mathrm{c}}$ \\ ${ }^{a}$ Brown University, Division of Engineering, Providence, USA $;{ }^{b}$ Department of Mechanical \\ Engineering, University of California, Santa Barbara, California, USA; ${ }^{c}$ Department of Applied \\ Physics, University of Groningen, AG Groningen, The Netherlands
}

(Received 14 January 2008; final version received 29 June 2008)

\begin{abstract}
The monotonic and cyclic crack growth rate of cracks is strongly influenced by the microstructure. Here, the growth of cracks emanating from pre-cracked micron-scale elastic particles and growing into single crystals is investigated, with a focus on the effects of (i) plastic confinement due to the elastic particle and (ii) elastic modulus mismatch between the reinforcement and matrix phases. Due to the small sizes of the particles and cracks, plasticity in the ductile crystal is modelled using a 2D discrete dislocation plasticity framework wherein dislocations are modelled as line singularities in an isotropic elastic isotropic material. Crack growth is modelled using a cohesive surface. Calculations reveal a threshold for fatigue crack growth and a transition to Paris power-law behavior, both depending on the existence of the elastic particle and the modulus mismatch. For a matched-modulus particle, the threshold is reduced by $25 \%$ and is attributed to slip blockage by the particle. For a high-modulus particle, the threshold is reduced by $50 \%$ due to the enhanced stress intensity factor caused by elastic mismatch and due to some slip blockage. However, crack growth halts after some amount of crack advance due to the decreasing effect of elastic mismatch and slip blocking as the crack moves away from the particle. The broad results here are compared with experimental observations in the literature, and are consistent in a number of respects. These results show that fatigue crack growth from micron-scale particles is strongly influenced by plasticity size effects, elastic mismatch, and particle constraints on plastic flow, all of which are captured within a discrete dislocation plasticity framework.
\end{abstract}

Keywords: discrete dislocations; cracked particle; crack growth; defects in solids

\section{Introduction}

A wide variety of structural alloys contain brittle particles dispersed in a ductile metallic matrix. While such particles are sometimes added for structural purposes, more often they arise during processing. These particles, or inclusions, are often undesirable byproducts of the material fabrication because fracture can originate by particle cracking followed by propagation into the surrounding ductile matrix. In particular, under cyclic loading

\footnotetext{
*Corresponding author. Email: William_Curtin@brown.edu
} 
conditions, cracks formed during processing or during the initial stages of loading will grow and ultimately lead to failure of the structure or component. Figure 1 shows one example of such fatigue crack growth from a particle in an Al-7075 alloy. The factors governing the propagation of a crack from a brittle particle into a surrounding ductile phase are thus of considerable practical importance for applications of many structural metals.

As a result of the problem's importance, computational and modelling studies of crack growth across an interface have been carried out under both monotonic and cyclic loading conditions, e.g. [1-8]. Some studies consider the materials on each side of the interface to deform plastically but with a mismatch in flow strength [9-11] while other studies consider one phase elastic and the other plastic [12]. Heterogeneity in material properties affects crack growth because: (i) the mismatch in material properties affects the near-crack tip stress distribution when the crack tip is near the interface; and (ii) there is typically a mismatch in the crack growth resistance of the phases on either side of the interface [13]. While the focus in previous studies has mainly been on crack growth across or along an interface, our aim is to model the situation depicted in Figure 1 where a crack initiates in a brittle particle and then grows into a ductile matrix. Our interest is in understanding the role of constraints on plastic flow due to the particle and the effect of elastic mismatch on the crack growth, both in the limit of micron-scale particles where plasticity size effects are known to play an important role.

Typically fatigue calculations are carried out within a framework where a fatigue crack growth law, e.g. a Paris law or a Coffin-Manson law, is postulated a priori and the emphasis is on calculating the driving force. While such laws can be calibrated for bulk materials through a suite of experimental studies under controlled loading situations and for long cracks, the resulting laws may not apply in other situations such as near an interface and/or for microstructurally small cracks, in which the plastic fields may be modified due to constraint and size effects. For instance, Gall et al. [8] use a continuum cyclic plasticity model to compute plastic strain ranges ahead of a cracked particle, but average over a size smaller than $1 \mu \mathrm{m}$ in front of the crack to obtain the driving force used in a subsequent crack growth law. While providing some guidance, the applicability of the assumed constitutive behavior is questionable. To account differences between small and large scales, continuum models have been developed to move from small cracks to large cracks $[14,15]$, but such models introduce additional parameters to characterize the fatigue behavior, which require extensive calibration and employ assumptions whose origins lie in concepts emerging from continuum plasticity.

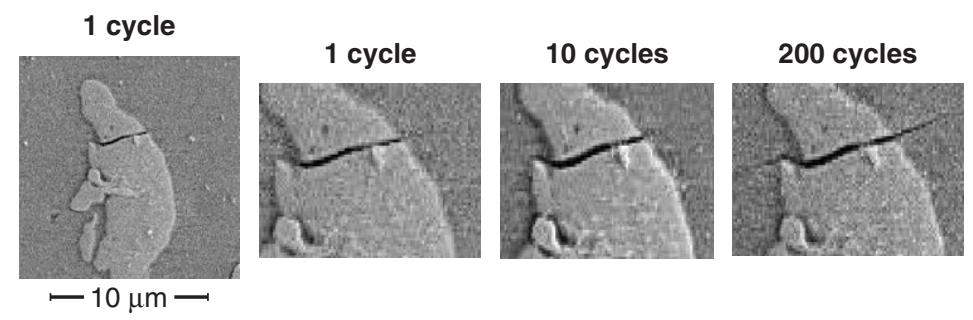

Figure 1. Fatigue crack initiating from a micron-scale particle and growing into the $\mathrm{Al}$ matrix in $\mathrm{Al}$ 7075 (Courtesy of Northrop-Grumman Corporation). 
Recent work has avoided assumptions of continuum plasticity by basing the plastic deformation on the creation, motion, and annihilation of dislocations. These discrete dislocation (DD) models have been shown to predict a host of phenomena, such as sizeeffects in yielding, fracture, sliding, indentation, and polycrystals [16-22], not accounted for in conventional continuum plasticity. In particular, DD studies of fatigue crack growth have been carried out $[17,18,24-27]$ that provide insight and guidance into fatigue crack growth problems where continuum plasticity and standard fatigue laws may be inapplicable. Most importantly for our current work is that these DD analyses predict that fatigue crack growth arises naturally under cyclic loading conditions without introducing additional parameters. The DD studies of fatigue in single-crystal materials have shown the existence of a threshold, a Paris power-law regime, striations, scaling with material properties and a short-crack regime [17,18,24].

Here, we use discrete dislocation plasticity to study monotonic and fatigue crack growth in a situation where both microstructure and micron-scale cracks exist, thus combining simultaneously elastic mismatch effects, interface effects, and small-crack effects. Specifically, we analyze the growth of a crack from an initially-cracked elastic particle into a ductile single crystal matrix. Plastic flow in the matrix arises from the motion of discrete dislocations following a set of constitutive rules discussed below. The fracture properties of the ductile material are embedded in a cohesive surface constitutive relation that permits only straight crack growth [23]. A key aspect of the formulation is that the plastic stress-strain response, the evolution of any dislocation structure, and the crack initiation and growth in the ductile matrix, are outcomes of the solution of the boundary value problem. We emphasize that the only distinction between an analysis of monotonic crack growth and crack growth under cyclic loading conditions is in the time dependence of the remote loading; the underlying material is identical. These features are common to prior DD studies of fatigue. The aim of the analyses here is to obtain insight into the effects of microstructural heterogeneity, through the presence of a cracked particle, on fatigue crack growth behavior. We find that the presence of a particle generally accelerates fatigue relative to the same crack in a single crystal, due to both elastic mismatch and plastic constraint effects, as discussed subsequently.

\section{Formulation}

We consider a planar single crystal reinforced by an elastic particle containing an initial crack, as sketched in Figure 2a. The formulation and numerical method follow those in Cleveringa et al. [28] and Deshpande et al. [17,24], except that here the elastic mismatch between the particle and the surrounding matrix gives rise to a polarization stress term.

The crystal matrix is taken to be elastically isotropic with Young's modulus $E_{\mathrm{m}}=70 \mathrm{GPa}$ and Poisson's ratio $v_{m}=0.33$, representative values for aluminium. The crystal matrix has three slip systems, with slip planes at an angle $\phi^{(\alpha)}, \alpha=1,2,3$ to the $x_{1}$-axis, with $\phi^{(1)}=60^{\circ}, \phi^{(2)}=-60^{\circ}$ and $\phi^{(3)}=0^{\circ}$, as shown in Figure 2a. Potential slip planes are spaced by $100 b$ where $b$ is the Burgers vector, $b=0.25 \mathrm{~nm}$. The particle is also taken to be elastically isotropic with Young's modulus $E_{\mathrm{p}}$ and Poisson's ratio $v_{p}=0.33$. Initially, the three slip systems are free of mobile dislocations, but dislocations are nucleated from point sources, randomly distributed with a density of $20 / \mu \mathrm{m}^{2}$. 


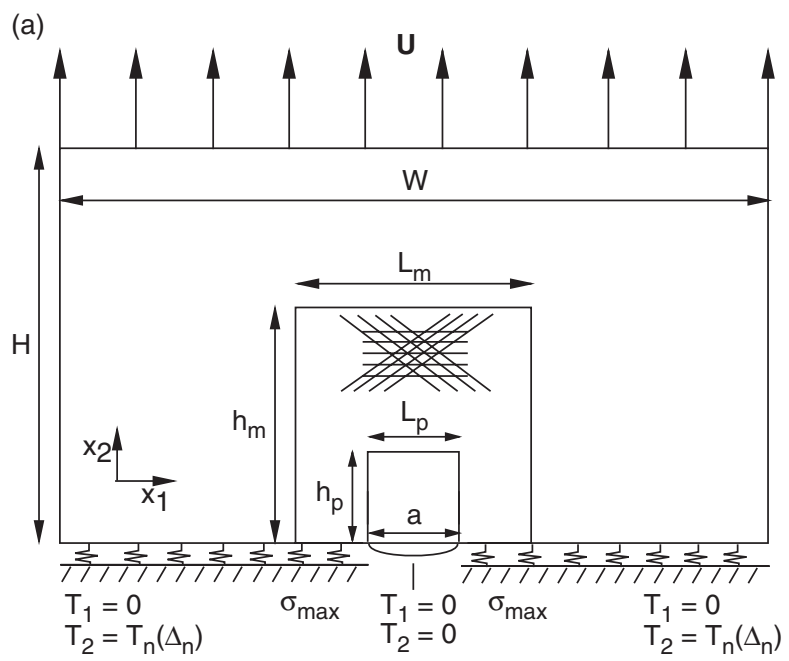

(b)

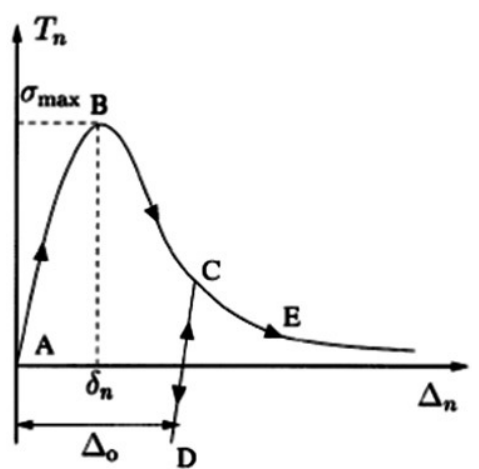

(c)

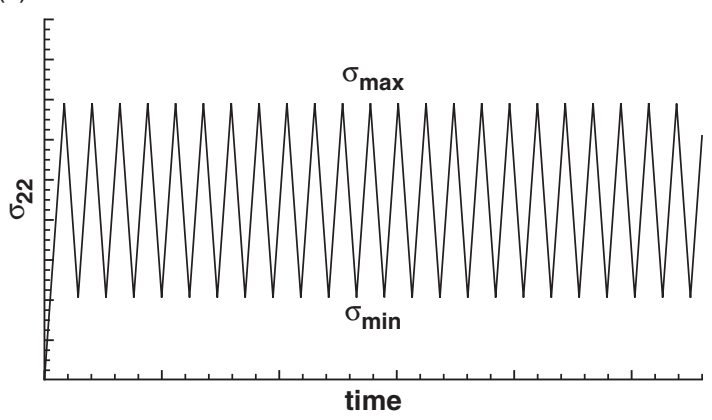

Figure 2. (a) Sketch of the analyzed specimen with the imposed boundary conditions. (b) Schematic of the cohesive relation employed. (c) Schematic of the applied stress versus time.

The dislocation sources mimic Frank-Read sources by nucleating a dipole when the Peach-Koehler force on the source exceeds the value $\tau_{\text {nuc }} b$ during a period of time $t_{\text {nuc }}$; here $\tau_{\text {nuc }}=20 \mathrm{MPa}$ and $t_{\text {nuc }}=10 \mathrm{~ns}$. The long-range elastic interactions between dislocations are accounted for directly in the boundary value problem solution. Shortrange interactions enter through a set of constitutive rules, of the type suggested by Kubin et al. [29], for dislocation glide, annihilation, and pinning by obstacles. The glide speed is taken to be linearly related to the Peach-Koehler force with a drag coefficient $B=10^{-4} \mathrm{Pas}$, a representative value for several fcc crystals [30]. Dislocations of opposite sign annihilate each other when they come within a critical distance of $L_{e}=6 \mathrm{~b}$. There is a random distribution of 50 point obstacles per $\mu \mathrm{m}^{2}$, which represent either small precipitates on the slip plane or forest dislocations on out-of-plane slip systems. These obstacles pin dislocations until the Peach-Koehler force attains the obstacle strength $b \tau_{\text {obs }}$, where $\tau_{\text {obs }}=60 \mathrm{MPa}$. For computational convenience, dislocation sources and obstacles are restricted to a process window of dimensions $L_{m} \times h_{m}$ around the cracked particle with $L_{m}=50 \mu \mathrm{m}$ and $h_{m}=50 \mu \mathrm{m}$ (see Figure 2a). 
The stress and displacement fields are obtained by superposition as described in [31]. The fields are written as

$$
\sigma_{i j}=\tilde{\sigma}_{i j}+\hat{\sigma}_{i j}, \quad \epsilon_{i j}=\tilde{\epsilon}_{i j}+\hat{\epsilon}_{i j}, \quad u_{i}=\tilde{u}_{i}+\hat{u}_{i},
$$

where the $(\sim)$-fields are the singular fields associated with each individual dislocation. The ( ) fields give rise to tractions $\tilde{T}_{i}$ and displacements $\tilde{u}_{i}$ on the boundary of the body. The ( $\left.{ }^{\wedge}\right)$ fields are the image fields that correct for the actual boundary conditions. The ( ${ }^{\wedge}$ ) fields are non-singular in the region of interest and are obtained by a finite element solution to a linear elastic boundary value problem.

The stress and strain fields are related by

$$
\sigma_{i j}= \begin{cases}L_{i j k l}^{m} \epsilon_{k l} & \text { in } V_{m} \\ L_{i j k l}^{p} \epsilon_{k l} & \text { in } V_{p},\end{cases}
$$

where $V_{m}$ denotes the volume occupied by the matrix and $V_{p}$ the volume occupied by the particle. Throughout $V=V_{m}+V_{p}$, the dislocation stress field $\tilde{\sigma}_{i j}$ is related to the strain field $\tilde{\epsilon}_{k l}$ by

$$
\tilde{\sigma}_{i j}=L_{i j k l}^{m} \tilde{\epsilon}_{k l}
$$

and hence

$$
\hat{\sigma}_{i j}= \begin{cases}L_{i j k l}^{m} \hat{\epsilon}_{k l} & \text { in } V_{m} \\ L_{i j k l}^{p} \hat{\epsilon}_{k l}+\left(L_{i j k l}^{p}-L_{i j k l}^{m}\right) \tilde{\epsilon}_{k l} & \text { in } V_{p} .\end{cases}
$$

The polarization stress term, $\left(L_{i j k l}^{p}-L_{i j k l}^{m}\right) \tilde{\epsilon}_{k l}$, vanishes for homogeneous elastic materials; i.e. for $L_{i j k l}^{p} \equiv L_{i j k l}^{m}$. Since $\tilde{\sigma}_{i j}$ satisfies equilibrium throughout $V$, $\hat{\sigma}_{i j}$ satisfies

$$
\frac{\partial \hat{\sigma}_{i j}}{\partial x_{j}}=0
$$

in $V$ with the boundary conditions,

$$
\hat{u}_{i}=u_{i}^{0}-\tilde{u}_{i} \quad \text { on } S_{u}, \hat{T}_{i}=T_{i}^{0}-\tilde{T}_{i} \quad \text { on } S_{T},
$$

where $S_{u}$ being that part of the boundary on which displacements $u_{i}^{0}$ are prescribed, $S_{T}$ being that part of the boundary on which tractions $T_{i}^{0}$ are prescribed, with $T_{i}=\sigma_{i j} n_{j}$ and $n_{j}$ is the outward normal to the surface. Equations (5) and (6) together with the constitutive relation Equation (4) give a boundary value problem for the ( ${ }^{\wedge}$ )-fields. The ( $\left.{ }^{\wedge}\right)$-fields are smooth in $V$ and a finite element method is used to obtain a solution. The polarization stress in Equation (4) is computed in FEM as an expensive (since longrange) body force.

The specific boundary value problem analyzed is sketched in Figure 2a. A rectangular block of height $2 H$ and width $W$ contains a centrally-located particle of height $2 h_{p}$ and width $L_{p}$. We use $W=100 \mu \mathrm{m}, H=500 \mu \mathrm{m}$, and $L_{p}=h_{p}=a=10 \mu \mathrm{m}$ with the particle between $-L_{p} / 2$ and $L_{p} / 2$ along the $x_{1}$-direction and between 0 and $h_{p}$ along the direction $x_{2}$. Symmetry about $x_{2}=0$ is assumed so the region analyzed occupies $-W / 2 \leq x_{1} \leq W / 2$ and $0 \leq x_{2} \leq H$. This block is subjected to tensile loading through a prescribed displacement rate $\dot{U}(t)$ on the top surface, which is taken to be shear free so that

$$
\dot{u}_{2}^{0}=\dot{U}(t), \dot{T}_{1}=0 \quad \text { on } x_{2}=H,
$$


where ( ) denotes time differentiation. The imposed stress $\sigma$ is then calculated as

$$
\sigma=\frac{1}{W} \int_{-W / 2}^{W / 2} T_{2}\left(x_{1}, H\right) \mathrm{d} x_{1}
$$

and, under cyclic loading conditions, has the time variation sketched in Figure $2 \mathrm{c}$ and the lateral sides are traction free.

In the calculations here, the particle is taken to be completely cracked so that the initial crack length is $a=L_{p}$. For a homogeneous elastic material, the applied stress $\sigma$ is related to the mode-I stress intensity factor as [32],

$$
K_{\mathrm{I}}=\sigma \sqrt{\pi a / 2}
$$

Corrections due to the finite ratio of $a / W=0.1$ are negligible. We note that Equation (9) does not give the local crack tip $K_{\mathrm{I}}$ in an elastically inhomogeneous material, but we nevertheless employ this definition to quantify the level of loading.

Crack growth is then modelled through a cohesive surface along $x_{2}=0$ in the matrix $\left(\left|x_{2}\right| \geq a / 2\right.$ ). Along the cohesive surface $T_{1}=0$ (from symmetry) while $T_{2}$ has the universal binding form [33]

$$
T_{2}\left(\Delta_{2}\right)=-\sigma_{\operatorname{coh}} \frac{\Delta_{2}}{\delta_{n}} \exp \left(-\frac{\left(\Delta_{2}-\delta_{n}\right)}{\delta_{n}}\right)
$$

where $\Delta_{2}=2 u_{2}\left(x_{1}, 0\right), \sigma_{\text {coh }}$ is the normal cohesive strength, and $\delta_{n}$ is a characteristic length. For a homogeneous elastic material and for monotonic loading, the work of separation is given by $\phi_{n}=\exp (1) \sigma_{\mathrm{coh}} \delta_{n}$ and is related to a reference stress intensity factor $K_{0}$ by

$$
K_{0}=\sqrt{\frac{E_{\mathrm{m}} \phi_{n}}{1-v_{m}^{2}}} .
$$

Under mode-I loading conditions, crack growth in an elastic solid initiates at $K_{\mathrm{I}} / K_{0}=1$ [13]. Unless otherwise specified the cohesive properties were taken as $\sigma_{\mathrm{coh}}=0.70 \mathrm{GPa}$ and $\delta_{n}=2 b$ giving a work of fracture $\phi_{n} \approx 1.0 \mathrm{~J} / \mathrm{m}^{2}$ and $K_{0} \approx 0.280 \mathrm{MPa} \sqrt{m}$. Associated with these parameters is a characteristic cohesive zone size $\delta_{c} \approx E_{m} \phi / \sigma_{c o h}^{2}$, which is $\approx 50 \mathrm{~nm}$ for the parameters used here. Since $\delta_{c} / a \ll 1$, the problems studied here are in the regime of small scale yielding. In addition, the numerical mesh size near the crack tip needs to resolve the length $\delta_{c}$ and so we use a mesh of size $17 \mathrm{~nm}$.

A cohesive relation that accounts for the irreversibility of separation is modelled by specifying unloading from and reloading to the monotonic cohesive function, as illustrated in Figure 2b [34]. Unloading from point $\mathrm{C}$ takes place along path $\mathrm{CD}$, with stiffness

$$
\frac{\partial T_{2}}{\partial \Delta_{2}}=-\frac{\exp (1) \sigma_{\mathrm{coh}}}{\delta_{n}} .
$$

During the reloading, the traction increases along DC and then follows the original softening curve BCE. This is a phenomenological cohesive relation introduced to model the effects of irreversibility arising from the formation of an oxide layer [34]. Under continued cyclic loading conditions, the permanent opening $\Delta_{0}$ (see Figure $2 \mathrm{~b}$ ) grows, but only up to a value $\Delta_{s}=4 \mathrm{~nm}$ which is a representative value for the oxide layer thickness on aluminium under ambient conditions [35]. 


\section{Results}

The presence of the elastic particle influences crack growth due to the effects of: (i) the modulus mismatch on the driving force for crack growth; (ii) the change in the evolution of plasticity in the vicinity of the crack tip; and (iii) the blocking of slip at the particle-matrix interface. Here, these effects are investigated separately and sequentially by considering the three cases summarized in Table 1: (i) material A, an initially-cracked single crystal, which should thus show only small crack effects; (ii) material B, a particle with the same elastic properties as the matrix, $E_{\mathrm{p}}=E_{\mathrm{m}}$ and $v_{p}=v_{m}$ but no dislocation activity in the particle, which should show the effects of slip blocking only; and (iii) material $\mathrm{C}$, a particle that is elastically stiffer than the matrix, $E_{\mathrm{p}}=5 E_{\mathrm{m}}$ but with $v_{p}=v_{m}$, which should show the effects of modulus mismatch and slip blocking acting together. To explore the influence of the specific distribution of sources and obstacles on the response, calculations were carried out for two sets of random distributions having the same densities of sources and obstacles but different specific spatial positions and referred to as, for instance, Material $B^{(j)}$ for $(j=0,1)$.

\subsection{Elastic response}

To calibrate our subsequent results on crack growth into plastic materials, we first investigate crack growth into an elastic matrix with no dislocations. These calculations demonstrate the effects of modulus mismatch and crack size versus particle size on the crack growth behavior, which will then be modified when plasticity in the matrix is permitted. For large cracks $a \gg L_{p}$, the critical stress intensity for crack growth should be independent of the particle properties.

Figure 3 shows the normalized stress intensity for crack growth (i.e. the so-called R-curve) as a function of the crack size $a$ for several values of the elastic modulus ratio. For a modulus ratio of unity, the crack begins to grow at $K_{\mathrm{I}} / K_{0} \approx 0.935$ but after a short amount of growth reaches a value of $K_{\mathrm{I}} / K_{0} \approx 0.985$. The onset at 0.935 is attributed to truncating the cohesive relation behind the initial crack tip location and the saturation value of 0.985 , just slightly below 1.0 , is due to mesh resolution in the cohesive zone. For particle elastic moduli exceeding that of the matrix, crack growth occurs well below $K_{\mathrm{I}} / K_{0}=0.985$, with the onset of fracture at $K_{\mathrm{I}} / K_{0}=0.68$ for $E_{p} / E_{m}=2$ and $K_{\mathrm{I}} / K_{0}=0.48$ for $E_{p} / E_{m}=5$. An uncracked high modulus particle would carry higher stresses than the matrix and thus, when cracked, increases the local crack tip stress intensity $K_{\mathrm{I}}^{(\text {loc) }}$ and lowers the applied stress intensity $K_{\mathrm{I}}$ needed to start fracture. The stress intensity for fracture increases rapidly with increasing initial crack size, relative to the particle size, for cracks up to $\approx a / L_{p}=1.2$, after which there is a slower increase to the asymptotic value of 0.99 . The effect of the cracked particle is thus most significant within $\approx 20 \%$ of the

Table 1. The three materials analyzed.

\begin{tabular}{lcc}
\hline Material & $E_{p} / E_{m}$ & Particle \\
\hline A & 1 & No \\
B & 1 & Yes \\
C & 5 & Yes \\
\hline
\end{tabular}


particle diameter. Thus, elastic modulus mismatch alone provides a significant decrease in fracture resistance and this will also manifest itself in the plastic response under monotonic and cyclic loading.

The above results allow for a calculation of the local stress intensity factor $K_{I}^{(\text {loc })}$ as a function of the crack size $a$ or crack extension $\Delta a$ as follows. The local stress intensity factor can be written as $K_{\mathrm{I}}^{(\mathrm{loc})}=f(a) K_{\mathrm{I}}$ where $f(a)$ is a function of crack length. For a homogeneous elastic material in small scale yielding, $f(a)=1$ and so fracture in the single-crystal material occurs when $K_{\mathrm{I}}^{(\mathrm{loc})}=K_{\mathrm{I}}=K_{\mathrm{Ic}} \approx 0.985 K_{0}$, independent of crack length $a$. With elastic mismatch, the local stress intensity factor differs from the remote stress intensity factor but at fracture $K_{\text {Ic }}^{(\text {loc }}=0.985 K_{0}$ must still hold. Thus, the critical applied stress intensity factor $K_{\mathrm{Ic}}(\Delta a)$ shown in Figure 3 satisfies $0.985 K_{0}=f(\Delta a) K_{\mathrm{Ic}}(\Delta a)$. Solving for $f(\Delta a)$ and substituting, we find the normalized local stress intensity factor in terms of the critical applied stress intensity factor as $K_{\mathrm{I}}^{(\mathrm{loc})} / K_{0}=0.985 K_{\mathrm{I}} / K_{\mathrm{Ic}}(\Delta a)$. Figure 3 for $K_{\mathrm{Ic}}(\Delta a)$ thus provides a master curve for relating the local and applied intensity factors.

\subsection{Monotonic loading}

A uniform displacement rate $\dot{U}(t) / H=100 / \mathrm{s}$ was imposed, chosen to reduce the computing time required. Under monotonic loading, it was previously found that varying the loading rate by two orders of magnitude did not qualitatively change the crack growth behavior, although a strong tendency was found for increased plastic deformation at lower loading rates [36].

The predicted $K_{\mathrm{I}} / K_{0}$ versus crack extension are shown in Figure $4 \mathrm{a}, \mathrm{b}$. Here and subsequently the crack tip location is taken to be the point along the cohesive surface where $\Delta_{2}=\delta_{n}$. In Figure 4a, b, $\Delta a_{r}$ is the crack growth in the $+x_{1}$-direction and $\Delta a_{l}$ is the crack growth in the $-x_{1}$-direction. In all three cases, the amount of crack growth is

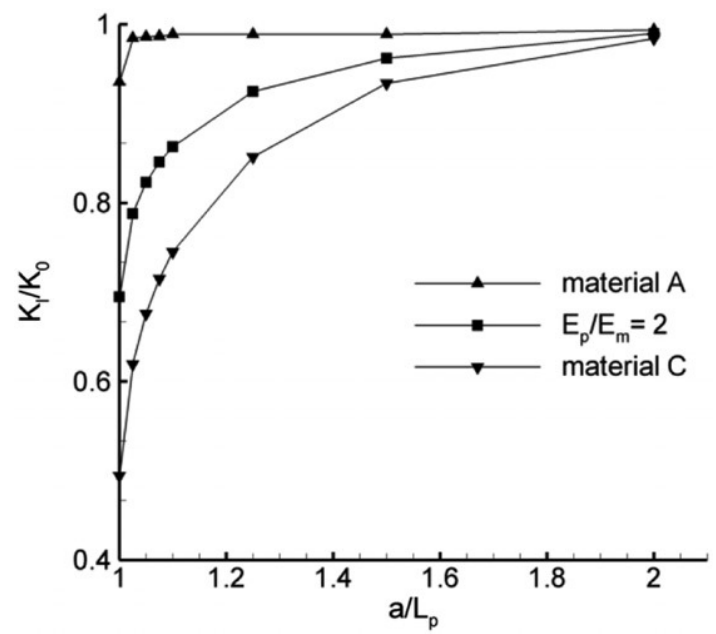

Figure 3. Normalized stress intensity for the onset of fracture as a function of initial crack size $a$ for several values of the elastic modulus ratio. 
different for the two crack tips. One reason for this asymmetry is simply that the distribution of sources and obstacles is not symmetric about $x_{1}=0$ and another is due to the extreme sensitivity of the dislocation dynamics to small perturbations [37]. For the single crystal, material A, crack growth only occurs in the $-x_{1}$-direction over the loading range shown. However, the values of $K_{\mathrm{I}} / K_{0} \approx 1.20$ at which crack growth initiates at the left crack tip are in good agreement with previous values [17]. For material B, where the presence of the particle blocks slip but with no modulus mismatch, crack growth occurs at both crack tips, initiating first on the left at $K_{\mathrm{I}} / K_{0} \approx 0.98-1.02$ and subsequently on the right at $K_{\mathrm{I}} / K_{0} \approx 1.11-1.26$. In material $\mathrm{C}$, where there is a modulus mismatch, the crack growth initiation values are $K_{\mathrm{I}} / K_{0} \approx 0.63$ and $K_{\mathrm{I}} / K_{0} \approx 0.55-0.72$ for the left and right crack tips, respectively. Thus, both the change in dislocation structure and the stress concentration induced by an elastic mismatch between the particle and the matrix promote the initiation of crack growth.

Normalizing the onset of crack growth in the plastic material by the value obtained in the elastic material (Figure 3) helps to highlight the role of slip blocking by the particle. For material $\mathrm{A}$, the ratio is $1.2 / 0.94=1.28$, for material $\mathrm{B}$ the ratios for the left and right crack tips are, on average, $\approx 1.06$ and $\approx 1.30$, respectively. For material $\mathrm{C}$, the ratios for the left and right crack tips are, on average, both about 1.31. These results suggest that elastic stress concentrations dominate the material response, with slip blocking playing a lesser role. In addition, slip blocking appears more important in driving crack growth for the matched-modulus particle as compared to the higher modulus particle, which responds similarly to the single-crystal material when the elastic stress concentration is normalized out.

The dislocation distributions for materials $\mathrm{A}^{(0)}, \mathrm{B}^{(0)}$ and $\mathrm{C}^{(0)}$ at $K_{\mathrm{I}} / K_{0}=1.07$ are shown in Figure 5, along with contours of the opening stress $\sigma_{22}$. In Figure 5a no particle is present, but for comparison purposes the region occupied by the particle in materials $\mathrm{B}$ and $\mathrm{C}$ is marked by dashed lines. The stress above the crack is relaxed and dislocations are

(a)

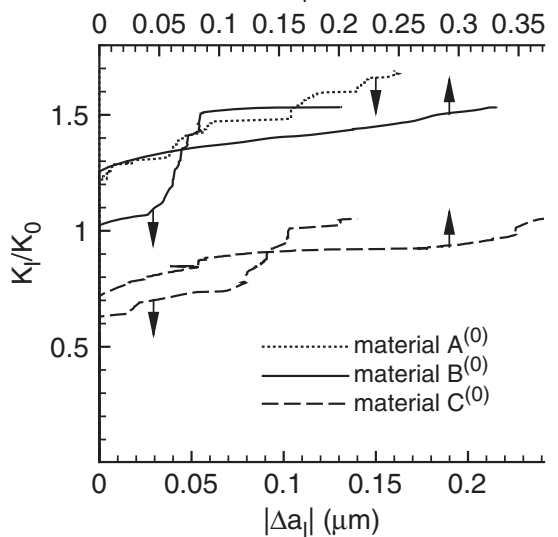

(b)

$\Delta \mathrm{a}_{\mathrm{r}}(\mu \mathrm{m})$

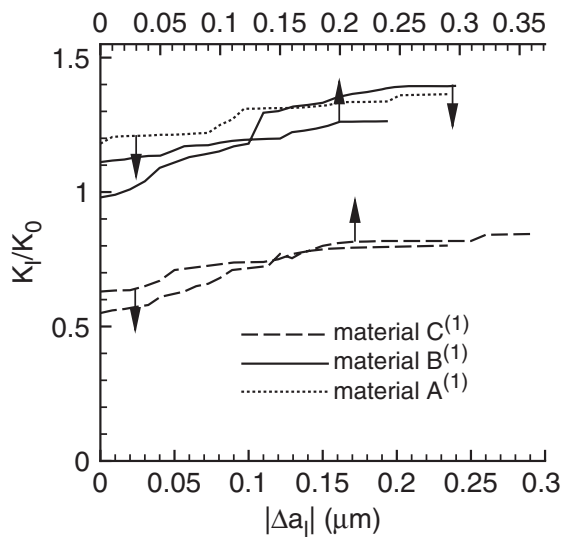

Figure 4. Normalized stress intensity $K_{\mathrm{I}} / K_{0}$ versus crack extension under monotonic loading. (a) Materials $\mathrm{A}^{(0)}, \mathrm{B}^{(0)}$ and $\mathrm{C}^{(0)}$; (b) Materials $\mathrm{A}^{(1)}, \mathrm{B}^{(1)}$ and $\mathrm{C}^{(1)}$. The superscripts (0) and (1) refer to realizations 1 and 2 , respectively. 
(a)

$$
\begin{gathered}
\sigma_{22} / \tau_{\text {nuc }}{ }_{-0.2} \\
\end{gathered}
$$
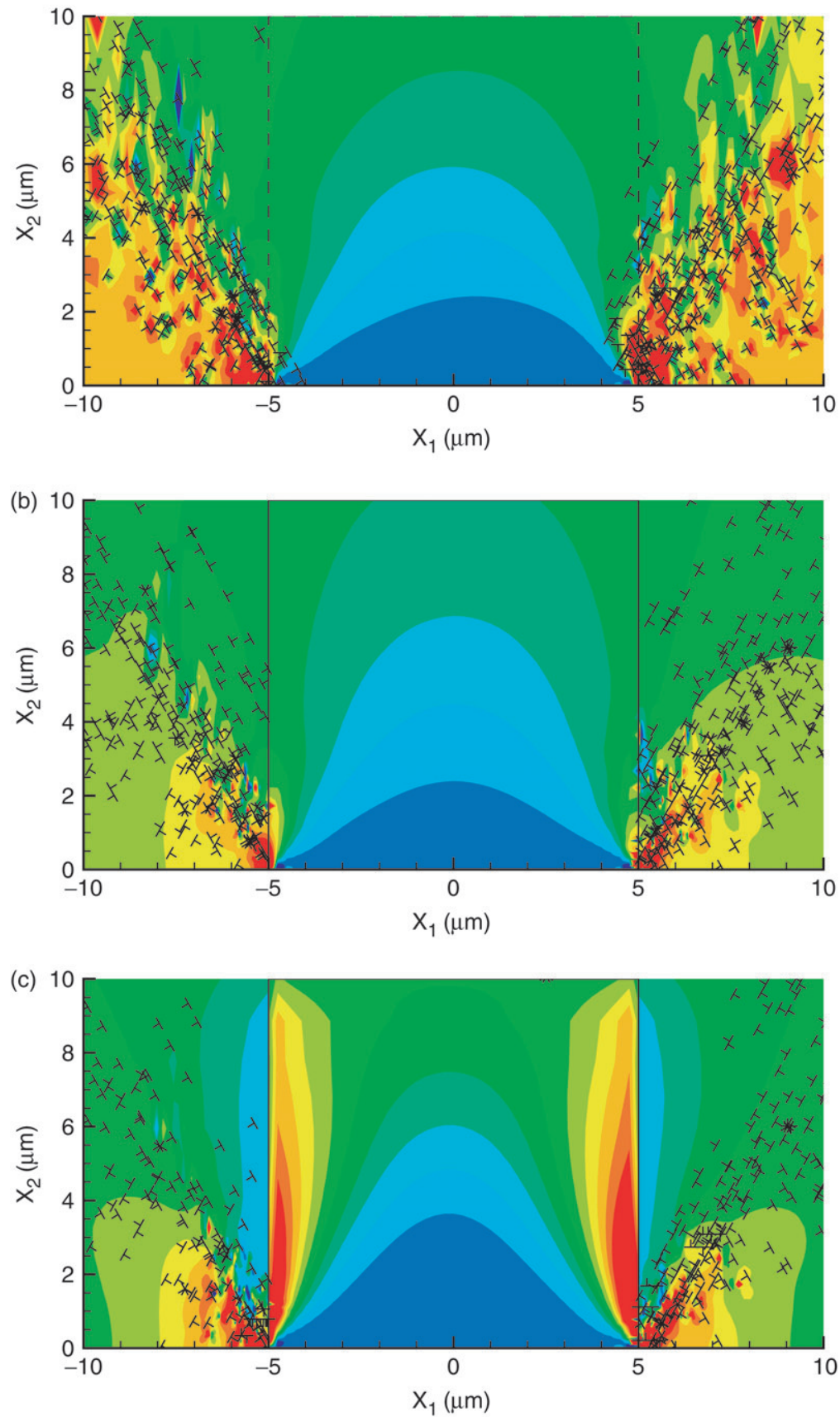

Figure 5. (Color online). Contours of normalized normal stress, $\sigma_{22} / \tau_{\text {nuc }}$, and dislocation distributions under monotonic loading at $K_{\mathrm{I}} / K_{0}=1.07$. (a) Material $\mathrm{A}^{(0)}$ (b) Material $\mathrm{B}^{(0)}$ (c) Material $\mathrm{C}^{(0)}$. The superscript $(0)$ refers to realization 1. 
present in the region above the crack. The difference between the stress distribution and dislocation structure at the two crack tips is evident. Evidence for the sector structure of the stress field as given by the continuum slip solution of Rice [38] can be seen. When a particle is present and dislocation glide is blocked, Figure $5 \mathrm{~b}$, the same stress distribution is obtained as in the single crystal, albeit with slightly higher stress gradients in the matrix near the two crack tips. With an elastic mismatch between the particle and the matrix, Figure $5 \mathrm{c}$, high stresses develop along the particle-matrix interface. In this case, the sector stress field structure is lost and the stress concentration in the particle increases and the stress gradients in the matrix near the two crack tips is greater. Moreover, with the elastic mismatch, more dislocations accumulate at the particle-matrix interface than when there is no elastic mismatch.

\subsection{Fatigue loading}

The same three sets of material parameters are used in the cyclic loading calculations as in the monotonic loading calculations in Section 3.2. The only difference is in the time dependence of the applied displacement $U(t)$, which has the form sketched in Figure 2c. All calculations are carried out with a displacement rate $\dot{U}_{0}=100 / \mathrm{s}$ and thus the variation of the cyclic loading amplitude is obtained by increasing or decreasing the frequency of loading between $3.3 \mathrm{MHz}$ and $8 \mathrm{MHz}$ to obtain the desired range of cyclic loading amplitudes. The applied loading is characterized by the stress intensity factor $K_{\mathrm{I}}$, with the stress varied between $\sigma_{\min }$ and $\sigma_{\max }$ and the corresponding stress intensity factor range $\Delta K_{\mathrm{I}}=K_{\max }-K_{\min }$. The load ratio, $R=\sigma_{\min } / \sigma_{\max }$ is fixed at 0.3 .

The DD calculations show fatigue crack growth above a threshold value of $\Delta K$. The crack growth shows a range of behaviors, and we first comment qualitatively. Figure 6 shows the computed crack advance, $\Delta a$, versus the number of cycles for $\Delta K_{\mathrm{I}}$ near the

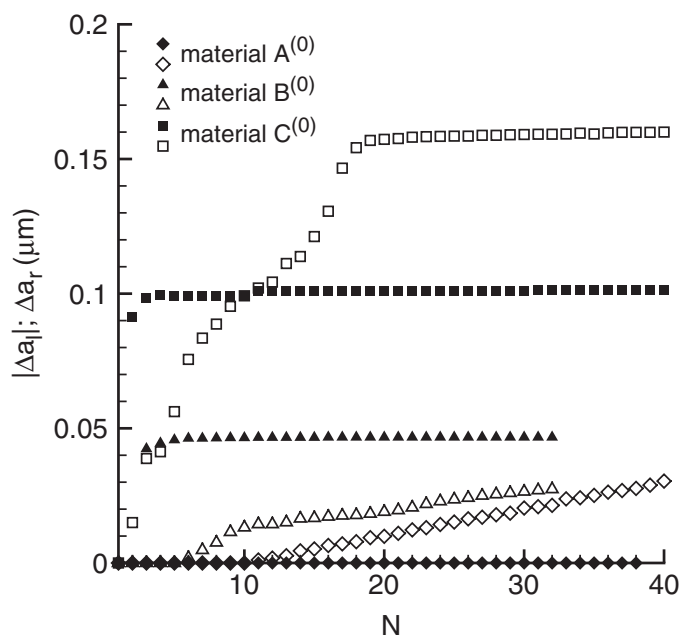

Figure 6. Crack advance versus number of cycles. The filled symbols are related to the left crack tip and unfilled symbols are related to the right crack tip. $\Delta K_{\mathrm{I}} / K_{0}=0.775,0.595$ and 0.395 for materials $\mathrm{A}^{(0)}, \mathrm{B}^{(0)}$ and $\mathrm{C}^{(0)}$, respectively. The superscript $(0)$ refers to realization 1. 
fatigue threshold found for each material. As for monotonic loading, the crack advance is not symmetric, but it differs from the monotonic loading case. Although crack growth to the left occurs at lower $K_{I}$ values under monotonic loading, under cyclic loading no crack advance occurs to the left in material $\mathrm{A}^{(0)}$ while some growth does occur in materials $\mathrm{B}^{(0)}$ and $\mathrm{C}^{(0)}$, but stopping after eight cycles and $\Delta a_{l} \mid \approx 0.05 \mu \mathrm{m}$ in material $\mathrm{B}^{(0)}$ and after 12 cycles and $\Delta a_{l} \mid \approx 0.10 \mu \mathrm{m}$ in material $\mathrm{C}^{(0)}$. Crack growth to the right, more difficult under monotonic loading, occurs more readily under cyclic loading. In material $\mathrm{A}^{(0)},\left|\Delta a_{r}\right|$ begins to increase after 10 cycles at a nearly constant rate. In material $\mathrm{B}^{(0)},\left|\Delta a_{r}\right|$ begins to increase after seven cycles at a slightly faster rate than for material $\mathrm{A}^{(0)}$, slowing to a lower rate after 10 cycles. In material $\mathrm{C}^{(0)},\left|\Delta a_{r}\right|$ shows two distinct regimes: (i) during the first 18 cycles, $\left|\Delta a_{r}\right|$ increases at a rate close to $0.008 \mu \mathrm{m} /$ cycle; and (ii) between cycles 19 and 40 , $\left|\Delta a_{r}\right|$ the rate decreases to a very low rate of about $10^{-4} \mu \mathrm{m} /$ cycle.

The normalized opening stress, $\sigma_{22} / \tau_{\text {nuc }}$, and dislocation distribution are shown in Figure 7 in a region around the cracked particle. For material $\mathrm{A}^{(0)}$, dislocations can glide into the material above the crack and relax the stress field. For materials $B^{(0)}$ and $C^{(0)}$, dislocation glide is blocked in the particle, which gives rise, even with no elastic mismatch, to an increased stress concentration above the crack tip that increases with increasing particle modulus. The different stress and dislocation distributions in the vicinity of the left and right crack tips, which are largely due to the different source and obstacle locations, can also be seen in Figure 7. In comparison to Figure 5, the dislocation distribution under cyclic loading is more concentrated along a $60^{\circ}$ slip plane near the right crack tip and along a $120^{\circ}$ slip plane near the left crack tip.

\subsection{Fatigue crack growth rate}

The crack growth rate $\log (\mathrm{d} a / \mathrm{d} N)$ versus the normalized loading $\log \left(\Delta K_{\mathrm{I}} / K_{0}\right)$ for $R=0.3$ is shown in Figure 8 a for materials $\mathrm{A}^{(j)}, \mathrm{B}^{(j)}$ and $\mathrm{C}^{(j)}$ with $j=0,1$, for crack growth on the right side. The crack growth rate is an average of the crack advance over the number of cycles computed. For materials $\mathrm{A}^{(j)}$ and $\mathrm{B}^{(j)}$ the average is over at least 10 cycles for $\Delta K_{\mathrm{I}}$ values above the threshold and over 20 cycles for near-threshold values of $\Delta K_{\mathrm{I}}$. As noted above, for material $\mathrm{C}$, the crack growth rates drop dramatically after crack extension exceeds a critical amount $\Delta a_{\text {crit }}$ and so the values of $\mathrm{d} a / \mathrm{d} N$ in Figure 8 a for materials $\mathrm{C}^{(0)}$ and $\mathrm{C}^{(1)}$ are averages over the number of cycles needed for the crack to grow to $\Delta a_{\text {crit }}$. The fatigue threshold is estimated using the procedure in Deshpande et al. [24] wherein calculations are carried out at decreasing values of $\Delta K_{\mathrm{I}}$ until the crack growth rate $\mathrm{d} a / \mathrm{d} N$ is less than or equal to $10^{-2} \mu \mathrm{m} /$ cycle (10 times larger than used in [24]). The fatigue threshold is then taken as the average of the last two values of the applied $\Delta K_{\mathrm{I}}$. Above the threshold, the crack growth rates are fit to a Paris power-law [39] of the form

$$
\frac{\mathrm{d} a}{\mathrm{~d} N}=C_{I}\left(\frac{\Delta K_{\mathrm{I}}}{K_{0}}\right)^{m},
$$

where $m$ is the Paris exponent and $C_{I}$ a constant. The fatigue threshold is reduced in the presence of a particle, and further reduced with an increased modulus mismatch. The thresholds are $\approx 0.78 K_{0}$ for material $\mathrm{A}, \approx 0.59 K_{0}$ for material $\mathrm{B}$, and $\approx 0.40 K_{0}$ for material $\mathrm{C}$, with only a small difference seen for the two distributions of dislocation sources and obstacles used in the calculations. The Paris exponent decreases when 
(a)

$$
\sigma_{22} / \tau_{\text {nuc }}
$$

$\begin{array}{lllllllllll}-0.20 & -0.10 & 0.00 & 0.24 & 0.47 & 0.69 & 0.91 & 1.13 & 1.36 & 1.58 & 1.80\end{array}$
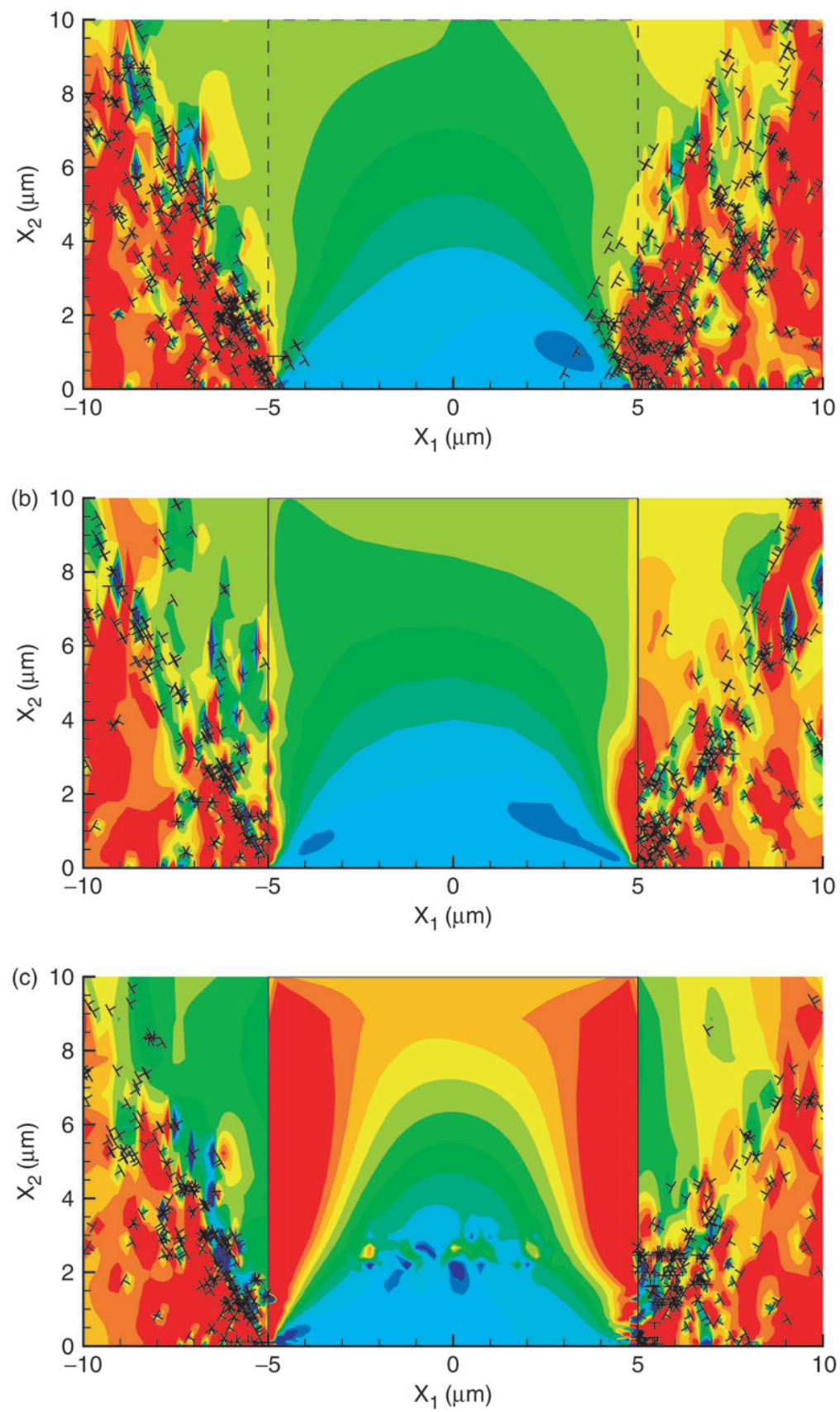

Figure 7. Contours of normalized normal stress, $\sigma_{22} / \tau_{\text {nuc }}$, and dislocation distributions. (a) Material $\mathrm{A}^{(0)}$ with $\Delta K_{\mathrm{I}} / K_{0}=0.775$. (b) Material $\mathrm{B}^{(0)}$ with $\Delta K_{\mathrm{I}} / K_{0}=0.595$. (c) Material $\mathrm{C}^{(0)}$ with $\Delta K_{\mathrm{I}} / K_{0}=0.395$. 

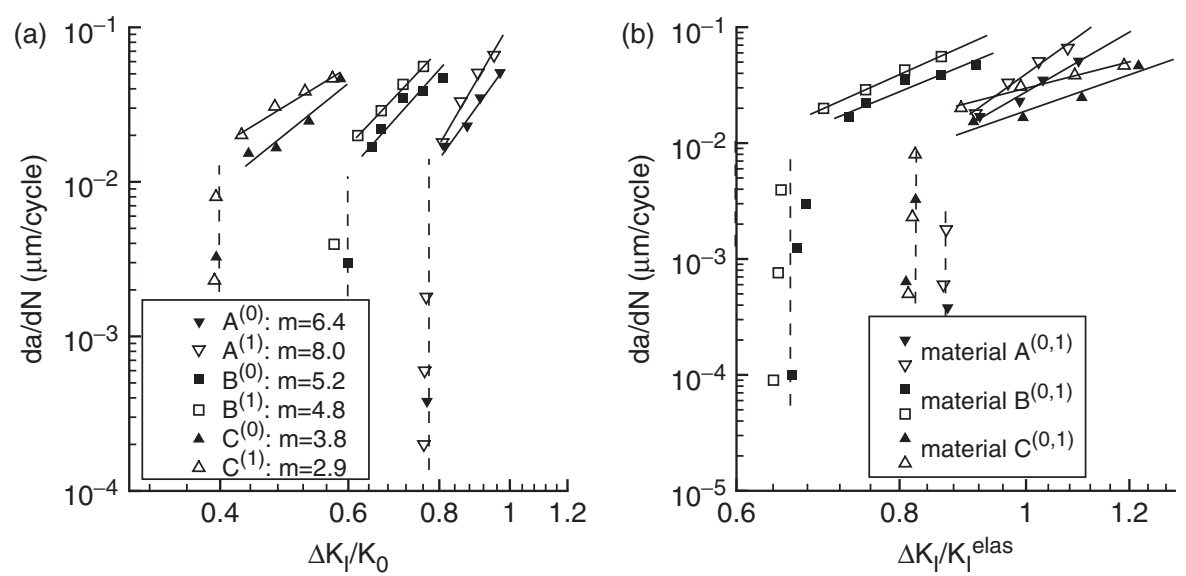

Figure 8. (a) Cyclic crack growth rate versus $\Delta K_{\mathrm{I}} / K_{0}$ for the mode I cyclic loading. For materials $\mathrm{A}^{0,1}$ and $\mathrm{B}^{0,1}$, the crack growth rate represents an average of the crack advance over 10 and 20 cycles for the low and high value of $\Delta K_{\mathrm{I}} / K_{0}$, respectively. For materials $\mathrm{C}^{0,1}$, the crack growth rate represents an average of the crack advance over the number of cycles needed for the crack to reach the critical distance. (b) Cyclic crack growth rate versus $\Delta K_{\mathrm{I}} / K_{\mathrm{I}}^{\text {elastic }}$ for the mode I cyclic loading. Filled symbols: material $\mathrm{A}^{(0)}, \mathrm{B}^{(0)}, \mathrm{C}^{(0)}$; open symbols: material $\mathrm{A}^{(1)}, \mathrm{B}^{(1)}, \mathrm{C}^{(1)}$.

a particle is present, and furthermore decreases with increasing mismatch, as indicated by Figure 8a. There is a somewhat greater sensitivity of the crack growth in the power-law regime to the specific realization which, although not large in magnitude, leads to a significant variation in the Paris exponent $m$. Keeping in mind that the crack in material C stops after a critical distance, these results show that a particle reduces the threshold for fatigue but decreases the rate of the crack growth rate.

We summarize salient values for the various material responses in Table 2. For cyclic loading at the fatigue threshold, the maximum stress intensity factor $K_{\max }^{\text {th }}$ is smaller than the stress intensity factor $K_{\mathrm{I}}$ obtained for the monotonic loading, and larger than the elastic value $K_{\mathrm{I}}^{\text {elastic }}$ for Materials A and C but smaller than $K_{\mathrm{I}}^{\text {elastic }}$ for Material B. The dislocations thus shield the crack tip under monotonic loading, but the dislocation structures formed under cyclic loading provide less shielding. The net shielding remains positive for Materials A and $\mathrm{C}$, because the threshold remains above $K_{\mathrm{I}}^{\text {elastic }}$, but is actually negative for Material B (anti-shielding). Net anti-shielding in fatigue has also been seen by Pippan and Weinert [40].

We now evaluate the fatigue results to attempt a decoupling of elastic and plastic effects on the fatigue crack growth. To do so, we first normalize the fatigue crack growth data against the critical stress intensity for crack growth in the elastic matrix problem (e.g. Figure 3), $\Delta K_{\mathrm{I}} / K_{\mathrm{I}}^{\text {elastic }}$, as shown in Figure $8 \mathrm{~b}$. Since $K_{\mathrm{I}}^{\text {elastic }} \approx K_{0}$, the results for materials $\mathrm{A}$ and $\mathrm{B}$ are changed only slightly. However, the $\mathrm{d} a / \mathrm{d} N$ versus $\Delta K_{\mathrm{I}}$ curves for material $C^{(j)}$ shift upward substantially, with the normalized fatigue threshold $\Delta K_{\mathrm{I}}^{\text {th }} / K_{\mathrm{I}}^{\text {elastic }}$ of material $C^{(j)}$ being comparable to that of material $A^{(j)}$. These results again indicate that slip blocking gives an anti-shielding effect for both monotonic and fatigue crack growth and that elastic mismatch increases the local stress intensity at the crack tip but the dislocation structures formed by the slip blocking and image stress fields cause shielding of the crack tip under both monotonic and cyclic loading conditions. 
Table 2. Numerical results for crack growth from a cracked particle, under monotonic loading for an elastic matrix and elastic-plastic one and under cyclic loading. The 'index' $j$ refers to the realization number.

\begin{tabular}{lcccccr}
\hline & Monotonic & & \multicolumn{3}{c}{ Cyclic } \\
\cline { 6 - 7 } Materials & $K_{\mathrm{I}}^{\text {elastic }} / K_{0}$ & $K_{\mathrm{I}} / K_{0}$ & & $\Delta K_{\mathrm{I}}^{\text {th }} / K_{0}$ & $K_{\max }^{\text {th }} / K_{0}$ & $\mathrm{~m}$ \\
\hline $\mathrm{A}^{(j)}$ & 0.94 & $\gg 1.22$ & & 0.78 & 1.10 & $6-8$ \\
$\mathrm{~B}^{(j)}$ & 0.94 & $1.03-1.26$ & & 0.59 & 0.83 & $4-6$ \\
$\mathrm{C}^{(j)}$ & 0.48 & $0.62-0.72$ & & 0.40 & 0.57 & $2-4$ \\
\hline
\end{tabular}
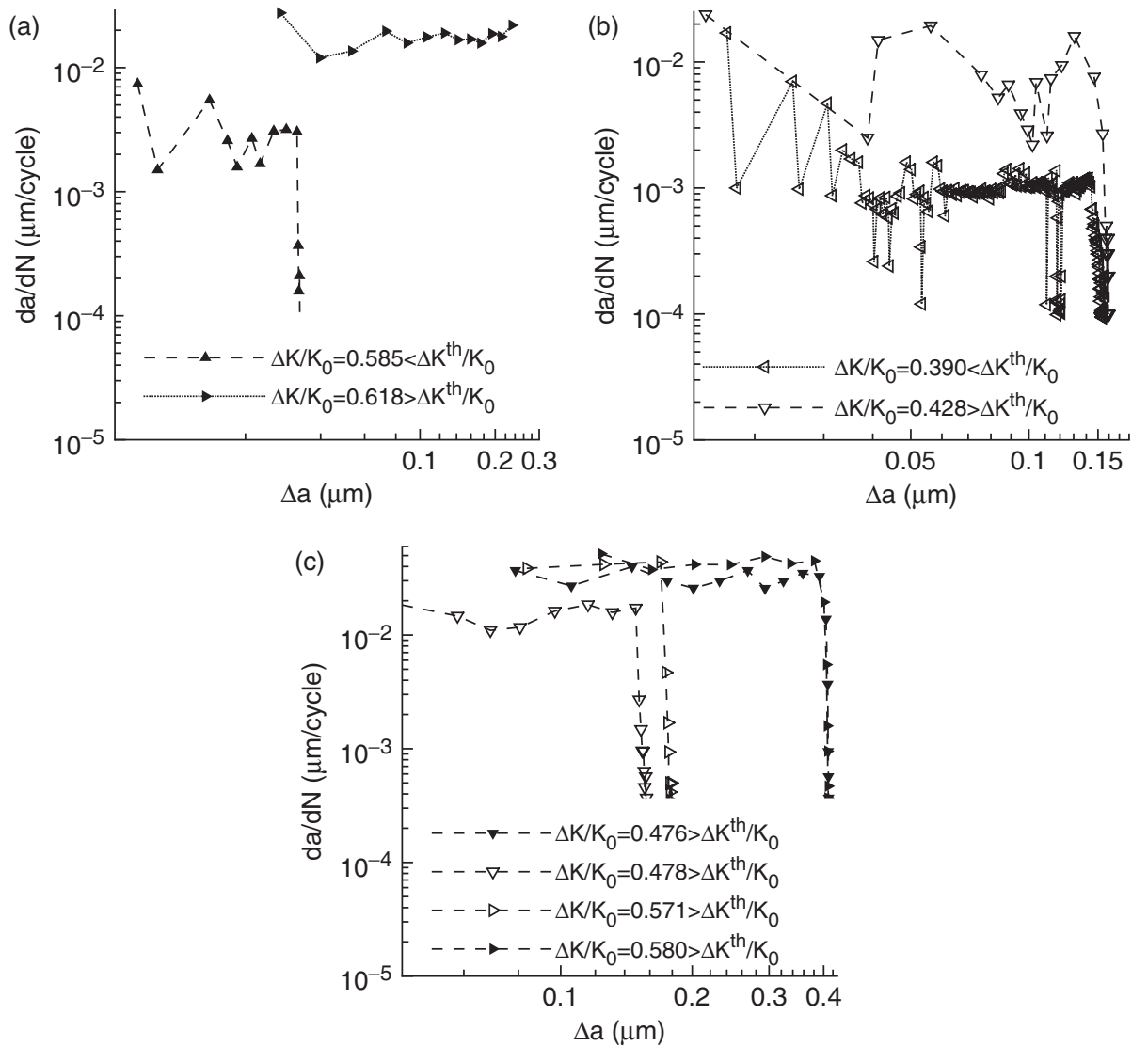

Figure 9. Crack growth rate versus crack advance for (a) Material $\mathrm{B}^{(0)}$ and (b) Material $\mathrm{C}^{(0)}$ around the fatigue threshold, and for (c) materials $\mathrm{C}^{(1)}$ (filled symbols) and $\mathrm{C}^{(0)}$ (unfilled symbols) for several values of $\Delta K_{\mathrm{I}}$ in the Paris regime.

Recall that crack growth in Materials B, C can be abruptly halted after growth out to a critical distance. We now examine this further. The crack growth rate as a function of the crack advance is plotted in Figure $9 a$ for material $B^{(0)}$, in Figure $9 b$ for material $C^{(0)}$ for values of the cyclic amplitude around the fatigue threshold, and in Figure $9 \mathrm{c}$ for materials 
$\mathrm{C}^{(0)}$ and $\mathrm{C}^{(1)}$ for several values of the cyclic amplitude $\Delta K_{\mathrm{I}}$ in the Paris power-law regime. Below the fatigue threshold, the crack growth rate versus crack advance is similar in materials $\mathrm{B}^{(0)}$ and $\mathrm{C}^{(0)}$ and, as noted previously, drops dramatically after a critical crack advance. For material $\mathrm{B}^{(0)}, \Delta a_{\text {crit }}=0.033 \mu \mathrm{m}$, while it is much larger at $\Delta a_{\text {crit }}=0.15 \mu \mathrm{m}$ in material $\mathrm{C}^{(0)}$ and $\Delta a_{\text {crit }}=0.40 \mu \mathrm{m}$ in material $\mathrm{C}^{(1)}$, showing sensitivity to the source/ obstacle distributions. Above the fatigue threshold, the crack growth is qualitatively different, still dropping dramatically in material $\mathrm{C}^{(0)}$ after a critical distance while being nearly constant in material $\mathrm{B}^{(0)}$.

We believe the reason that cracks stop growing is that the local stress intensity amplitude $\Delta K_{\mathrm{I}}^{\text {(loc) }}$ driving growth is decreasing with crack growth due to either changes in slip blocking or elastic mismatch effects, or both, and can decrease below a threshold value $\Delta K_{\mathrm{I}}^{\text {(loc)th }}$. Since for larger crack growth, we expect that the crack growth rates for Materials A, B, and $\mathrm{C}$ will converge, the existence of a unique threshold is not unreasonable, and so we consider $\Delta K_{\mathrm{I}}^{\text {(loc)th }}$ to be a material parameter. We envision that the $\Delta K_{\mathrm{I}}^{(\mathrm{loc})}$ is increased, relative to the applied $\Delta K_{\mathrm{I}}$, by (i) a slip blocking contribution $\Delta K_{\mathrm{I}}^{\mathrm{sb}}$ and (ii) the elastic stress concentration factor found previously for elastic fracture, $0.985 \Delta K_{\mathrm{I}} / \Delta K_{\mathrm{Ic}}(\Delta a)$, so that

$$
\Delta K_{\mathrm{I}}^{\text {(loc) }}=\frac{0.985 K_{0} \Delta K_{\mathrm{I}}}{\Delta K_{\mathrm{Ic}}(\Delta a)}+\Delta K_{\mathrm{I}}^{\mathrm{sb}}
$$

With a threshold $\Delta K_{\mathrm{I}}^{\text {(loc)th }}$, a crack loaded at $\Delta K_{\mathrm{I}}$ will halt after an amount of crack growth $\Delta a$ satisfying

$$
\Delta K_{\mathrm{I}}^{(\text {loc }) \text { th }}=0.985 K_{0} \Delta K_{\mathrm{I}} / \Delta K_{\mathrm{Ic}}(\Delta a)+\Delta K_{\mathrm{I}}^{\mathrm{sb}} .
$$

For material $\mathrm{A}$, the local and applied stress intensity amplitudes are equal, $\Delta K_{\mathrm{I}}^{(\text {loc })}=\Delta K_{\mathrm{I}}$, and so we can immediately estimate $\Delta K_{\mathrm{I}}^{(\text {loc)th }}=0.78 K_{0}$. Then, for material B with no elastic stress concentration, we have $\Delta K_{\mathrm{I}}^{\text {(loc)th }}=\Delta K_{\mathrm{I}}^{\text {th }}+\Delta K_{\mathrm{I}}^{\text {sb }}$ and so can estimate $\Delta K_{\mathrm{I}}^{\mathrm{sb}}=\Delta K_{\mathrm{I}}^{(\mathrm{loc}) \text { th }}-\Delta K_{\mathrm{I}}^{\mathrm{th}}=0.19 K_{0}$ since $\Delta K_{\mathrm{I}}^{\text {th }}=0.59 K_{0}$ for Material B. For material $\mathrm{C}$, both slip blocking and an elastic stress concentration play a role and lead to crack growth at lower applied loads but with halting of the crack after larger amounts of growth. For material $\mathrm{C}$ we account for the elastic stress concentration through the factor $f(a)$ and so can estimate the slip blocking as $\Delta K_{\mathrm{I}}^{\mathrm{sb}}=\Delta K_{\mathrm{I}}^{(\text {loc)th }}-0.985 K_{0} \Delta K_{\mathrm{I}} / \Delta K_{\mathrm{Ic}}(\Delta a)$ using the measured values of $\Delta K_{\mathrm{I}}$ and $\Delta a$ at which cracks stop. Table 3 summarizes the results of this analysis on Materials A, B, C. The estimated slip blocking for Material C is generally smaller than for Material B and decreases at higher applied $\Delta K_{\mathrm{I}}$. In one case, the estimated

Table 3. Summary of the numerical analysis. Lines 4-7 refer to materials $C^{(0,1)}$ in the Paris power-law regime as given in Figure 9c.

\begin{tabular}{lccccc}
\hline Material & $\Delta K_{\mathrm{I}} / K_{0}$ & $\Delta a(\mu \mathrm{m})$ & $K_{\mathrm{Ic}} / K_{0}$ & $\Delta K_{\mathrm{I}}^{(\text {loc)th }} / K_{0}$ & $\Delta K_{\mathrm{I}}^{(s b)} / K_{0}$ \\
\hline A & 0.78 & - & 0.985 & 0.78 & - \\
B & 0.59 & - & 0.985 & $(0.78)$ & 0.19 \\
C & 0.428 & 0.15 & 0.63 & $(0.78)$ & 0.11 \\
& 0.476 & 0.4 & 0.72 & $(0.78)$ & 0.13 \\
& 0.478 & 0.16 & 0.635 & $(0.78)$ & 0.04 \\
& 0.571 & 0.18 & 0.64 & $(0.78)$ & -0.10 \\
& 0.580 & 0.4 & 0.72 & $(0.78)$ & -0.01 \\
\hline
\end{tabular}


slip blocking becomes negative. This suggests that the slip blocking effect decreases as the crack grows more than a few tenths of a micron from the particle and/or is influenced by image stresses due to the particle-matrix elastic modulus mismatch. This limited influence of slip blocking is consistent with our discussion above for monotonic loading.

Overall, our results are reasonably interpreted through the consideration of a $\Delta K_{\mathrm{I}}^{\text {loc }}$ that is affected by:

(i) slip blocking, which gives an anti-shielding effect for small crack extension;

(ii) elastic mismatch, which gives a stress concentration that decreases with crack growth;

(iii) interactions between the above two factors, which influences the dislocation structure that develops, and is reflected in fluctuations of the estimates above.

The first two factors act to reduce the value of the applied $\Delta K_{I}$ necessary to initiate and continue fatigue crack growth, relative to a single crystal material.

\section{Discussion}

A number of prior studies exist on the role of particles in modifying fatigue crack growth in metals. Based on a continuum crystal plasticity model, Bruzzi et al. [41] estimated the state of stress within the matrix and incorporated the stress gradient along a predefined crack path into investigation of the effect of reinforcing particles on a small fatigue crack [42]. The existence of such a gradient of stress is important because it increases the driving force at the crack tip and thus accelerates the crack growth close to the interface $[12,43]$. We see the same effect here when there is elastic mismatch, while slip blocking may add an additional contribution. Jiang et al. [44] experimentally studied fatigue crack propagation normal to an elastically-matched but plastically mismatched bimetallic interface under a four-point bending condition. They observed fatigue crack acceleration over a few tenth of micrometers beyond the interface when the crack propagated from a hard (high yield stress) phase into a soft (lower yield stress) phase, followed by a reduced growth. A similar result was obtained numerically by Wang and Siegmund [12] using a cohesive framework to model crack propagation through an elastically-matched, plastically-mismatched interface. These results are qualitatively similar to our results for material $\mathrm{B}$, except that our hard phase is strictly elastic. In contrast to the continuum framework, however, we observe fatigue with maximum loads below the elastic fracture limit, indicating short-crack and discrete plasticity effects. We also observe stochastic effects such as asymmetry in the crack growth, and a sensitivity of the growth rates and loads to the distribution of obstacles and sources.

The fatigue threshold and the crack growth rate characterize the propagation of a crack during a fatigue experiment. From the literature, it appears that the only common feature is that fatigue crack growth behavior is different for single and multiphase materials, and that an increase or a decrease of the fatigue threshold is strongly dependent on the material properties [45]. Tanaka et al. [46] present results showing that $\Delta K_{\mathrm{I}}^{\text {th }}$ tends to be larger for reinforced as compared to unreinforced alloys at the maximum tensile stress level. Such an increase of the fatigue threshold in presence of reinforcements is also observed by Milan and Bowen [47] in the case of an 
Al matrix reinforced by different volume fraction of $\mathrm{SiC}$ particles. On the other hand, for $16 \%$ and $21 \% \mathrm{Al}_{2} \mathrm{O}_{3}$ particle reinforced aluminium alloys, Pippan and Weinert [40] conclude experimentally that the fatigue threshold of the particle reinforced alloy is smaller than the one obtained for the unreinforced material, in agreement with this study and with the trend in [40]. Literature data further suggest that the presence of reinforcements tends to increase the crack growth resistance (inverse of the Paris exponent) as compared non-reinforced material. Chen et al. [48] concluded that the crack growth rate decreases with increasing particle size. Milan and Bowen [47] characterized separately the crack growth resistance of unreinforced and reinforced aluminium alloys, concluding that the addition of $\mathrm{SiC}$ particles increases the fatigue crack growth resistance at near threshold and Paris power-law regimes. This latter effect of the reinforcement on the crack growth resistance is reproduced by the calculations presented in this study. The reduced Paris exponent may be attributable to the higher dislocation density close to the crack and particle in Material $\mathrm{C}$ as compared to Material B (Figure 7b,c) because an increase in plastic dissipation tends to decrease the Paris exponent in ductile materials [13].

\section{Conclusions}

Crack growth from small initially-cracked elastic particles into a ductile metal material has been analysed using discrete dislocation plasticity to characterize the inelastic deformation in the matrix at micron scales and using a cohesive model for fracture. Analysis of crack growth under fatigue loading reveals the following general features:

- The particle reduces the threshold value of $\Delta K$ and the Paris power-law exponent, relative to a single-crystal material.

- Slip blocking at the particle-matrix interface gives rise to a dislocation structure with increased anti-shielding relative to the single-crystal material.

- Stress concentrations due to elastic mismatch increase the local stress intensity factor further and thus reduce the applied stress intensity factor at which crack growth is observed.

- The elastic stress concentrations decay with increasing crack length, leading to halting of crack growth near and above the threshold value of $\Delta K$ after some critical distance of crack growth.

\section{Acknowledgements}

The authors gratefully acknowledge support of this work by Northrop-Grumman Corporation and by the National Science Foundation through the Materials Research Science and Engineering Center on Nano- and Micromechanics of Materials at Brown University, grant no. DMR-0520651.

\section{References}

[1] S. Suresh, Y. Sugimura and E.K. Tschegg, Scripta Metall. Mater. 27 (1992) p.1189.

[2] A. Romeo and R. Ballarini, Int. J. Solids Struct. 34 (1997) p.1307.

[3] D.H. Chen, Int. J. Fracture 88 (1997) p.19. 
[4] A.S. Kim, S. Suresh and C.F. Shih, Int. J. Solids Struct. 34 (1997) p.3415.

[5] T. Siegmund and N.A. Fleck, Int. J. Fracture 85 (1997) p.381.

[6] D. Wäppling, J. Gunnars and P. Ståhle, Int. J. Fracture 89 (1998) p.223.

[7] J.W. He, N. Li, M. Jia et al., Fatigue Fract. Eng. Mater. Struct. 12 (2002) p.171.

[8] K. Gall, M.F. Horstemeyer, B.W. Degner et al., Int. J. Fracture 108 (2001) p.207.

[9] Y. Sugimura, P.G. Lim, C.F. Shih et al., Acta Metall. Mater. 43 (1995) p.1157.

[10] R. Pippan and F.O. Riemelmoser, Comp. Mat. Sci. 13 (1998) p.108.

[11] M.T. Milan and P. Bowen, Int. J. Fatigue 25 (2003) p.649.

[12] B. Wang and T. Siegmund, Int. J. Plasticity 22 (2006) p.1586.

[13] S. Suresh, Fatigue of Materials, Cambridge University Press, Cambridge, 2000.

[14] D.L. McDowell, K. Gall, M.F. Horstemeyer et al., Eng. Fracture Mech. 70 (2003) p.49.

[15] Y. Xue, D.L. McDowell, M.F. Horstemeyer et al., Eng. Fracture Mech 74 (2007) p.2810.

[16] L. Nicola, Y. Xiang, J.J. Vlassak et al., J. Mech. Phys. Solids 54 (2006) p.2089.

[17] V.S. Deshpande, A. Needleman and E. Van der Giessen, Acta Mater. 51 (2003) p.1.

[18] V.S. Deshpande, A. Needleman and E. Van der Giessen, Acta Mater. 51 (2003) p.4637.

[19] A.C. Chng, M.P. O’Day, W.A. Curtin et al., Acta Mater. 54 (2006) p.1017.

[20] A. Widjaja, E. Van der Giessen, V.S. Deshpande et al., J. Mater. Res. 22 (2007) p.655.

[21] D.S. Balint, V.S. Deshpande, A. Needleman et al., Mater. Sci. Eng. A 400-401 (2005) p.186.

[22] D.S. Balint, V.S. Deshpande, A. Needleman et al., Model. Simul. Mater. Sci. Eng. 14 (2006) p.409.

[23] A. Needleman, J. Appl. Mech. 54 (1987) p.525.

[24] V.S. Deshpande, A. Needleman and E. Van der Giessen, Acta Mater. 50 (2002) p.831.

[25] F.O. Riemelmoser, R. Pippan and O. Kolednik, Comput. Mech. 20 (1997) p.139.

[26] F.O. Riemelmoser, P. Gumbsch and R. Pippan, Mater. Trans. JIM 42 (2001) p.2.

[27] A.J. Wilkinson, S.G. Roberts and P.B. Hirsch, Acta Mater. 46 (1998) p.379.

[28] H.H.M. Cleveringa, E. Van der Giessen and A. Needleman, J. Mech. Phys. Solids 48 (2000) p.1133.

[29] L.P. Kubin, G. Canova, M. Condat et al., Solid State Phenomena $23-24$ (1992) p.455.

[30] J. Philibert, in Dislocations et Deformation Plastique, P. Groh, L.P. Kubin and J.-L. Martin eds., Les Editions de Physique, Les Ulis, 1979, p.101.

[31] E. Van der Giessen and A. Needleman, Model. Simul. Mater. Sci. Eng. 3 (1995) p.689.

[32] H. Tada, P.C. Paris and G.R. Irwin, The Stress Analysis of Cracks Handbook, 3rd ed., ASME, New York, 2000.

[33] J.H. Rose, J. Ferrante and J.R. Smith, Phys. Rev. Lett. 47 (1981) p.675.

[34] V.S. Deshpande, A. Needleman and E. Van der Giessen, Acta Mater. 49 (2001) p.3189.

[35] T. Do, S.J. Splinter, C. Chen et al., Surf. Sci. 387 (1997) p.192.

[36] H.H.M. Cleveringa, E. Van der Giessen and A. Needleman, Mater. Sci. Eng. A: Struct. 317 (2001) p.37.

[37] V.S. Deshpande, A. Needleman and E. Van der Giessen, Scripta Mater. 45 (2001) p.1047.

[38] J.R. Rice, Mech. Mater. 6 (1987) p.317.

[39] P.C. Paris, M. Gomez and W.E. Anderson, Trend Eng 13 (1961) p.9.

[40] R. Pippan and P. Weinert, Phil. Mag. A 77 (1998) p.875.

[41] M.S. Bruzzi, P.E. McHugh, F. O'Rourke et al., Int. J. Plasticity 17 (2001) p.565.

[42] M.S. Bruzzi and P.E. McHugh, Int. J. Fatigue 26 (2004) p.795.

[43] R. Pippan, R.O. Flechisg and F.O. Riemelmoser, Mater. Sci. Eng. A A238 (2000) p.225.

[44] F. Jiang, Z.L. Deng, K. Zhao et al., Mater. Sci. Eng. A 356 (2003) p.258.

[45] N. Narasaiah and K.K. Ray, Int. J. Fatigue 28 (2006) p.891.

[46] K. Tanaka, Y. Akiniwa, K. Shimizu et al., Int. J. Fatigue 22 (2000) p.431.

[47] M.T. Milan and P. Bowen, J. Mater. Engng Performance 13 (2004) p.612.

[48] Z.Z. Chen, K. Tokaji and A. Minagi, J. Mater. Sci. 36 (2001) p.4893. 